\title{
Departures from LTE for neutral Li in late-type stars ${ }^{\star}$
}

\author{
K. Lind ${ }^{1}$, M. Asplund ${ }^{2}$, and P. S. Barklem ${ }^{3}$ \\ ${ }^{1}$ European Southern Observatory (ESO), Karl-Schwarzschild-Strasse 2, 85748 Garching bei München, Germany \\ e-mail: klind@eso.org \\ 2 Max-Planck-Institut für Astrophysik, Karl-Schwarzschild-Strasse 1, 85741 Garching bei München, Germany \\ 3 Department of Physics \& Astronomy, Uppsala University, Box 515, 75120 Uppsala, Sweden
}

Received 28 March 2009 / Accepted 17 May 2009

\begin{abstract}
We perform non-LTE calculations of lithium in late-type stars for a wide range of stellar parameters, including quantum mechanical cross-sections for collisions with neutral hydrogen and the negative hydrogen ion. Non-LTE abundance corrections for the lithium resonance line at $670.7 \mathrm{~nm}$ and the subordinate line at $610.3 \mathrm{~nm}$, are calculated using 1D MARCS model atmospheres spanning a grid $T_{\text {eff }}=[4000,8000] \mathrm{K}, \log g=[1.0,5.0]$, and $[\mathrm{Fe} / \mathrm{H}]=[0.0,-3.0]$, for lithium abundances in the range $A(\mathrm{Li})=[-0.3,4.2]$. The competing effects of ultraviolet over-ionization and photon losses in the resonance line govern the behaviour of the non-LTE effects with stellar parameters and lithium abundance. The size and sign of the non-LTE abundance corrections vary significantly over the grid for the $670.7 \mathrm{~nm}$ line, but are typically positive and below 0.15 dex for the $610.3 \mathrm{~nm}$, line. The new collisional data play a significant role in determining the abundance corrections.
\end{abstract}

Key words. stars: abundances - stars: late-type - line: formation

\section{Introduction}

Stellar lithium abundances are continuing to attract strong interest among the astronomical community. In particular, many studies are devoted to constraining and finding explanations for the famous "Spite plateau" of warm metal-poor halo stars, first discovered by Spite \& Spite (1982). For recent studies of lithium in halo field stars see e.g. Ryan et al. (2001), Charbonnel \& Primas (2005), Asplund et al. (2006), Bonifacio et al. (2007), and Hosford et al. (2009). To put constrainst on the primordial lithium abundance in the Universe, it is of fundamental importance to determine if and by how much the Li abundances increase with increasing metallicity on the Spite plateau. The behaviour of Li abundance with effective temperature is of equal importance, as it could lend support to the notion that atomic diffusion is acting in metal-poor stellar atmospheres. Lithium depletion through atomic diffusion has been suggested as a solution to the discrepancy between the Spite plateau abundance and the predicted value of the primordial lithium abundance (see e.g. Korn et al. 2007; Lind et al. 2009). To accurately infer both the mean lithium abundance in the halo and the abundance behaviour with metallicity and effective temperature, it is crucial to have a realistic description of the lithium line formation. Previous non-LTE analyses spanning a large stellar-parameter space (Carlsson et al. 1994; Pavlenko \& Magazzu 1996; Takeda \& Kawanomoto 2005) have shown that departures from LTE are generally small but significant at the required accuracy.

Barklem et al. (2003) applied quantum-mechanical calculations of cross sections for inelastic collisions with neutral hydrogen (Belyaev \& Barklem 2003; Croft et al. 1999) in

\footnotetext{
* Complete Tables 2 and 3 are only available in electronic form at the CDS via anonymous ftp to cdsarc.u-strasbg.fr (130.79.128.5) or via
}

http://cdsweb.u-strasbg.fr/cgi-bin/qcat? J/A+A/503/541
non-LTE analysis of the Sun and two metal-poor stars. Their findings point to a negligible influence on the statistical equilibrium of lithium from collisional bound-bound transitions with hydrogen, but a significant influence from charge transfer reactions, specifically mutual neutralization and ion-pair production $\left(\mathrm{Li}^{*}+\mathrm{H} \leftrightarrows \mathrm{Li}^{+}+\mathrm{H}^{-}\right)$. Other non-LTE investigations have relied on the classical Drawin recipes (Drawin 1968), as given by Steenbock \& Holweger (1984) and Lambert (1993), or the free electron model of Kaulakys (1985), for estimates of collisions with neutral hydrogen. None except Barklem et al. have included the influential charge transfer reaction.

We have extended the study by Barklem et al. (2003) to cover a large cool-star grid and calculate non-LTE abundance corrections for the lithium $670.7 \mathrm{~nm}(2 \mathrm{~s}-2 \mathrm{p})$ and $610.3 \mathrm{~nm}(2 \mathrm{p}-3 \mathrm{~d})$ lines.

\section{Setup}

We use the radiative-transfer code MULTI, version 2.3 (Carlsson $1986,1992)$ to perform non-LTE calculations. The model atom used includes the same 20 energy levels for neutral lithium as described in Carlsson et al. (1994), plus the Li II ground state. The highest considered level in Li I has principle quantum number $n=9$. We have used TOPbase data (Peach et al. 1988) for energy levels, oscillator strengths, and photo-ionization crosssections for levels with orbital quantum number $l \leq 3$. For the remaining levels, hydrogenic values are used. For the resonance line at $670.7 \mathrm{~nm}$, we adopt the oscillator strength $f=0.7468$, as calculated by Yan et al. (1998), and consider six hyperfine components in ${ }^{7} \mathrm{Li}$ (neglecting ${ }^{6} \mathrm{Li}$ ), with measured wavelengths given by Sansonetti et al. (1995). For the subordinate line at $610.3 \mathrm{~nm}$, we adopt $f=0.6386$, also determined by Yan et al., and account for the three fine-structure components with wavelengths determined by Lindgård \& Nielson (1977). For both 
Table 1. Wavelengths, oscillator strengths, and broadening data for the two considered lines.

\begin{tabular}{llllll}
\hline \hline \multicolumn{7}{c}{$2 \mathrm{~s}^{2} \mathrm{~S}-2 \mathrm{p}^{2} \mathrm{P}^{\mathrm{o}}$} \\
\multicolumn{7}{c}{${ }^{(a)} \Gamma=3.690 \times 10^{7}$} & ${ }^{(b)} \sigma=346$ & ${ }^{(c)} \alpha=0.236$ \\
\hline$\lambda[\mathrm{nm}]$ & $J_{1}$ & $J_{\mathrm{u}}$ & $f$ & $F_{1}$ & $F_{\mathrm{u}}$ \\
\hline 670.79080 & $1 / 2$ & $1 / 2$ & $1.037 \times 10^{-2}$ & 1 & 1 \\
670.79066 & $1 / 2$ & $1 / 2$ & $5.186 \times 10^{-2}$ & 1 & 2 \\
670.79200 & $1 / 2$ & $1 / 2$ & $3.112 \times 10^{-2}$ & 2 & 1 \\
670.79187 & $1 / 2$ & $1 / 2$ & $3.112 \times 10^{-2}$ & 2 & 2 \\
670.77561 & $1 / 2$ & $3 / 2$ & $1.245 \times 10^{-1}$ & 1 & $0,1,2$ \\
670.77682 & $1 / 2$ & $3 / 2$ & $1.245 \times 10^{-1}$ & 2 & $1,2,3$ \\
\hline \multicolumn{7}{c}{$2 \mathrm{p}^{2} \mathrm{P}^{\mathrm{o}}-3 \mathrm{~d}^{2} D$} \\
${ }^{(a)} \Gamma=1.055 \times 10^{8}{ }^{(b)} \sigma=837$ & ${ }^{(c)} \alpha=0.274$ \\
\hline$\lambda[\mathrm{nm}]$ & $J_{1}$ & $J_{\mathrm{u}}$ & $f$ \\
\hline 610.3538 & $1 / 2$ & $3 / 2$ & $6.386 \times 10^{-1}$ \\
610.3664 & $3 / 2$ & $3 / 2$ & $6.386 \times 10^{-2}$ \\
610.3649 & $3 / 2$ & $5 / 2$ & $5.747 \times 10^{-1}$ \\
\hline
\end{tabular}

(a) $\Gamma\left[\mathrm{rad} \mathrm{s}^{-1}\right]$ is the natural broadening parameter.

(b) $\sigma$ [a.u.] is the broadening cross-section for collisions with neutral hydrogen at relative velocity $v=10^{4} \mathrm{~m} \mathrm{~s}^{-1}$ (Anstee \& O'Mara 1995).

(c) $\alpha$ is the velocity dependence of $\sigma$.

lines, van-der-Waals-broadening parameters follow Anstee \& O'Mara (1995) and Barklem \& O'Mara (1997). Stark broadening is unimportant in the late-type atmospheres of interest here and is therefore neglected. Table 1 lists wavelenghts, oscillator strengths, and broadening data adopted for the $670.7 \mathrm{~nm}$ and $610.3 \mathrm{~nm}$ lines.

Cross-sections for collisional excitation by electrons are taken from Park (1971) and collisional ionization by electrons from Seaton (1962) as given by Allen (1976). We add rate coefficients for excitation and de-excitation from collisions with neutral hydrogen atoms according to Belyaev \& Barklem (2003) and Barklem et al. (2003), as well as charge transfer reactions with neutral hydrogen and the negative hydrogen ion according to Croft et al. (1999). The number abundance of the negative hydrogen ion is calculated by assuming LTE. Ionization by hydrogen atom impact is not included. For low-lying states at low collision energies, it is expected to be negligible compared to charge transfer reactions and excitation (see e.g. Krstić \& Schultz 2009).

A grid of 1D, LTE, opacity-sampling, MARCS model atmospheres (Gustafsson et al. 2008) is used in the analysis. Non-LTE computations for lithium as a trace element are performed in the plane-parallel approximation, for models with $T_{\text {eff }}=[4000,8000] \mathrm{K}, \log g=[1.0,5.0],[\mathrm{Fe} / \mathrm{H}]=[0.0,-3.0]$, for lithium abundances in the range $A(\mathrm{Li})=[-0.30,-4.20]$. The highest effective temperature is $5500 \mathrm{~K}$ for models with $\log g=1.0,6500 \mathrm{~K}$ for $\log g=2.0,7500 \mathrm{~K}$ for $\log g=3.0$, and $8000 \mathrm{~K}$ for $\log g \geq 4$.0. For models with $\log g \geq 3$.0, we adopted a microturbulence parameter $\xi_{\mathrm{t}}=1.0 \mathrm{~km} \mathrm{~s}^{-1}$ and $\xi_{\mathrm{t}}=2.0 \mathrm{~km} \mathrm{~s}^{-1}$, and for models with $\log g \leq 3.0$, we adopted $\xi_{\mathrm{t}}=2.0 \mathrm{~km} \mathrm{~s}^{-1}$ and $\xi_{\mathrm{t}}=5.0 \mathrm{~km} \mathrm{~s}^{-1}$. In total, 392 atmospheric models are included in the computations.

In the statistical equilibrium calculations, the background opacities include continuum and line opacities provided by the MARCS model atmospheres. MULTI thus calculates a lineblanketed photo-ionizing radiation field while solving for the statistical equilibrium of lithium. Based on the LTE and nonLTE equivalent widths obtained for the stellar-parameter grid, we subsequently define a non-LTE correction for each abundance point as the difference between its LTE lithium abundance
Table 2. Non-LTE abundance corrections and equivalent widths for the Li I $\lambda=670.7 \mathrm{~nm}$ line.

\begin{tabular}{llllllll}
\hline \hline $\begin{array}{l}T_{\text {eff }} \\
{[\mathrm{K}]}\end{array}$ & $\log g$ & {$[\mathrm{Fe} / \mathrm{H}]$} & $\begin{array}{l}\xi_{\mathrm{t}} \\
{\left[\mathrm{km} \mathrm{s}^{-1}\right]}\end{array}$ & $A(\mathrm{Li})_{\mathrm{LTE}}$ & $\Delta^{(a)}$ & $\begin{array}{l}W_{\lambda, \mathrm{LTE}} \\
{[\mathrm{pm}]}\end{array}$ & $\begin{array}{l}W_{\lambda, \text { NLTE }} \\
{[\mathrm{pm}]}\end{array}$ \\
\hline 4000 & 1.0 & -3.0 & 2.0 & -0.3 & 0.25 & 2.24 & 1.29 \\
4000 & 1.0 & -3.0 & 2.0 & 0.0 & 0.25 & 4.20 & 2.49 \\
4000 & 1.0 & -3.0 & 2.0 & 0.3 & 0.25 & 7.48 & 4.65 \\
4000 & 1.0 & -3.0 & 2.0 & 0.6 & 0.24 & 12.28 & 8.28 \\
4000 & 1.0 & -3.0 & 2.0 & 0.9 & 0.21 & 18.05 & 13.66 \\
4000 & 1.0 & -3.0 & 2.0 & 1.2 & 0.14 & 23.71 & 20.40
\end{tabular}

(a) $\Delta=A(\mathrm{Li})_{\text {non-LTE }}-A(\mathrm{Li})_{\mathrm{LTE}}$. The whole table can be retrieved in machine-readable format from the CDS.

and the non-LTE abundance that corresponds to the same equivalent width. The limiting equivalent widths for which corrections are given are set to $0.01 \mathrm{pm}$ and $100 \mathrm{pm}$. The equivalent width is obtained by numerical integration over the line profile, considering a spectral region that extends $\pm 9 \mathrm{~nm}$ from the line centre. For lines weaker than $50 \mathrm{pm}$ the numerical precision is better than $0.01 \mathrm{pm}$ whereas the equivalent widths of the strongest lines are determined to within $0.1 \mathrm{pm}$.

\section{Results}

The abundance corrections and LTE and non-LTE equivalent widths for each grid point are given in Table 2 for the $670.7 \mathrm{~nm}$ line, and in Table 3 for the $610.3 \mathrm{~nm}$ line. In the following we summarize the most important non-LTE effects and describe the size of the corrections over the cool-star grid. Because our results are in qualitative agreement with Carlsson et al. (1994), we refer the reader to this paper for a detailed explanation of the non-LTE line formation of lithium.

At $A(\mathrm{Li})<2$ for the coolest giants and $A(\mathrm{Li})<3$ for the hottest giants, the dominant non-LTE effect is over-ionization of neutral lithium, driven mainly by the super-thermal radiation field at $\lambda \lesssim 349.5 \mathrm{~nm}\left(J_{v}>B_{v}\right.$, where $J_{v}$ is the mean intensity and $B_{v}$ the Planck function), corresponding to the photoionization edge for the first excited level. This leads to smaller number populations of the ground ( $2 s$ ) and first excited (2p) states compared to LTE and in higher values of the line source functions. Both the effect on the source functions and the loss of line-opacity weaken the lines in non-LTE, making the abundance corrections positive. The corrections range from being close to zero for the hottest giants to a maximum +0.45 dex for cool, metal-rich giants for the $670.7 \mathrm{~nm}$ line. For the $610.3 \mathrm{~nm}$ line, the corrections are slightly smaller, $\leq+0.3$ dex.

In dwarfs, the $J_{v}-B_{v}$ excess is smaller in the $2 \mathrm{p}$ photoionization continuum and the radiation field is sub-thermal, $J_{v}<B_{v}$, in other influential continua, e.g. those corresponding to photo-ionization from $3 \mathrm{p}$ and $3 \mathrm{~d}$. In layers close to continuum optical depth unity, hot dwarfs have overpopulations in the ground state, which in combination with a decrease in the resonance-line source function strengthens the $670.7 \mathrm{~nm}$ line. Abundance corrections are therefore negative or approximately zero for this line for dwarfs hotter than $6000 \mathrm{~K}$.

At $A(\mathrm{Li})>2$ for the coolest stars and $A(\mathrm{Li})>3$ for the hottest stars, photon losses in the resonance line become apparent, driving recombination from Li II in both dwarfs and giants. This mainly affects the population of the ground state and to a lesser degree higher excited states. The resonance-line source function drops accordingly and the non-LTE corrections become less positive or more negative with increasing abundance over 
Table 3. Non-LTE abundance corrections and equivalent widths for the Li I $\lambda=610.3 \mathrm{~nm}$ line.

\begin{tabular}{llllllll}
\hline \hline $\begin{array}{l}T_{\text {eff }} \\
{[\mathrm{K}]}\end{array}$ & $\log g$ & {$[\mathrm{Fe} / \mathrm{H}]$} & $\begin{array}{l}\xi_{\mathrm{t}} \\
{[\mathrm{km} / \mathrm{s}]}\end{array}$ & $A(\mathrm{Li})_{\mathrm{LTE}}$ & $\Delta^{(a)}$ & $\begin{array}{l}W_{\lambda, \mathrm{LTE}} \\
{[\mathrm{pm}]}\end{array}$ & $\begin{array}{l}W_{\lambda, \mathrm{NLTE}} \\
{[\mathrm{pm}]}\end{array}$ \\
\hline 4000 & 1.0 & -3.0 & 2.0 & 0.0 & 0.17 & 0.02 & 0.02 \\
4000 & 1.0 & -3.0 & 2.0 & 0.3 & 0.17 & 0.05 & 0.03 \\
4000 & 1.0 & -3.0 & 2.0 & 0.6 & 0.17 & 0.09 & 0.06 \\
4000 & 1.0 & -3.0 & 2.0 & 0.9 & 0.17 & 0.19 & 0.13 \\
4000 & 1.0 & -3.0 & 2.0 & 1.2 & 0.17 & 0.37 & 0.25 \\
4000 & 1.0 & -3.0 & 2.0 & 1.5 & 0.17 & 0.73 & 0.49 \\
$\ldots$ & $\ldots$ & $\ldots$ & $\ldots$ & $\ldots$ & $\ldots$ & $\ldots$ & $\ldots$
\end{tabular}

(a) $\Delta=A(\mathrm{Li})_{\text {non-LTE }}-A(\mathrm{Li})_{\mathrm{LTE}}$. The whole table can be retrieved in machine-readable format from the CDS.

the whole grid. For metal-poor, extremely lithium-rich giants, the corrections can reach almost -1.0 dex.

Since the number population of the first excited state and the source function of the subordinate line are not as severely affected by the photon losses in the resonance line, the $610.3 \mathrm{~nm}$ abundance corrections for $A(\mathrm{Li})<4.0$ are approximately constant for fixed stellar parameters.

As described in Barklem et al. (2003), including hydrogen collisions strengthens the collisional coupling between singly ionized lithium and neutral lithium, especially through the charge transfer reaction involving $\mathrm{H}$ and $\mathrm{LiI}$ in the $3 \mathrm{~s}$ state and its inverse reaction. The number populations of low excited states increase when charge transfer is included, while the line source functions are unaffected. In turn, the abundance corrections become generally lower, i.e. less positive or more negative. Notice that the added collisions do not always have a thermalising effect, but can contribute to over-population of levels compared to LTE. The effect is illustrated in Fig. 1, for a selected metal-poor and solar-metallicity dwarf and giant. As seen in Fig. 1, the change in $A(\mathrm{Li})_{\text {non-LTE }}-A(\mathrm{Li})_{\text {LTE }}$ when including charge transfer is -0.12 dex for the metal-poor giant and $-0.06 \mathrm{dex}$ for the metal-poor dwarf.

While the charge transfer reaction has a significant influence on the statistical equilibrium of lithium, including bound-bound (and bound-free) transitions due to collisions with neutral hydrogen has no influence at all. However, this is not true if one relies on the classical Drawin recipe for estimates of the cross-section for those collisions. For comparison, Fig. 1 also shows the results obtained when neglecting charge transfer reactions but including excitation and ionization by neutral hydrogen according to Drawin's recipe, with the rate formula given by Lambert (1993) and with the scaling factor $S_{\mathrm{H}}=1.0$. Generally, the Drawin cross-sections are much higher than the quantum mechanical calculations by Belyaev \& Barklem. When adopting the higher classical rates, the ground state is more populated and the abundance corrections become lower, by $\sim-0.05$ dex, compared to the results obtained with quantum mechanical rates.

\section{Discussion}

We have compared our results for the lithium resonance line to Carlsson et al. (1994) abundance corrections for dwarfs and giants with $[\mathrm{Fe} / \mathrm{H}]=[-3.0,0.0]$ to Pavlenko \& Magazzu (1996) results for dwarfs and subgiants with solar metallicity and to Takeda \& Kawanomoto (2005) corrections for dwarfs and subgiants with $[\mathrm{Fe} / \mathrm{H}]=[0.0,-1.0]$ (see Fig. 3). To aid in the comparison we converted the LTE and non-LTE equivalent widths listed in Table 1 in Pavlenko \& Magazzu (1996) to abundance



Fig. 1. Non-LTE abundance corrections for $670.7 \mathrm{~nm}$ as functions of LTE lithium abundance for the indicated stellar parameters in the lower left corner of each plot, to be read as $T_{\text {eff }} / \log g /[\mathrm{Fe} / \mathrm{H}]$. Solid: including charge transfer reactions with hydrogen as well as bound-bound transitions due to collisions with neutral hydrogen. The cross-sections are calculated with quantum mechanics (Belyaev \& Barklem 2003; Croft et al. 1999). Dotted: neglecting charge transfer reactions, but including bound-bound transitions, calculated with quantum mechanics. Dashed: neglecting charge transfer reactions, but including hydrogen collisions for bound-bound and bound-free transitions. The cross sections are calculated with the classical Drawin recipe.

corrections. The largest difference is 0.12 dex between our results and those of Pavlenko \& Magazzu and 0.15 dex between our results and those of Takeda \& Kawanomoto, but generally the values agree quite well. The Carlsson et al. (1994) values agree with ours to within 0.15 dex for dwarfs and 0.20 dex for giants. Such differences are reasonable considering differences in the model atom and in the atmospheric models. Carlsson et al. use Gustafsson et al. (1975) MARCS models and Takeda \& Kawanomoto (2005) and Pavlenko \& Magazzu (1996) use Kurucz (1993) ATLAS9 models. The effects on the nonLTE abundance corrections when using MARCS models from Gustafsson et al. (1975) and Gustafsson et al. (2008) are illustrated in Fig. 2. Especially for the two giants, the choice of model atmosphere is important for the corrections. The newer models include more line opacity, causing a steeper temperature gradient in the upper part of the photosphere, which increases the ultraviolet $J_{v}-B_{v}$ excess and leads to more over-ionization. The use of newer model atmospheres thus leads to more positive or less negative abundance corrections and partly cancels with the effect of including hydrogen collisions (see Sect. 3).

Varying the microturbulence between $1 \mathrm{~km} \mathrm{~s}^{-1}$ and $2 \mathrm{~km} \mathrm{~s}^{-1}$ barely affects the abundance corrections (lower panels in Fig. 2), especially for low lithium abundances. In a giant star, the nonLTE abundance corrections become systematically higher when adopting a microturbulence of $5 \mathrm{~km} \mathrm{~s}^{-1}$ instead of $2 \mathrm{~km} \mathrm{~s}^{-1}$, the differences being especially significant when the line is strong (upper panels Fig. 2). However, the parameter still has some significance at low lithium abundances, when the formation of the line itself is unaffected by the microturbulence. This is because the choice of microturbulence influences the amount of line opacity included in the computations of the model atmosphere and consequently also the atmospheric temperature gradient, which in turn partly drives the departures from LTE.

Our results are valid within the assumptions of 1D model atmospheres in LTE and hydrostatic equilibrium. 


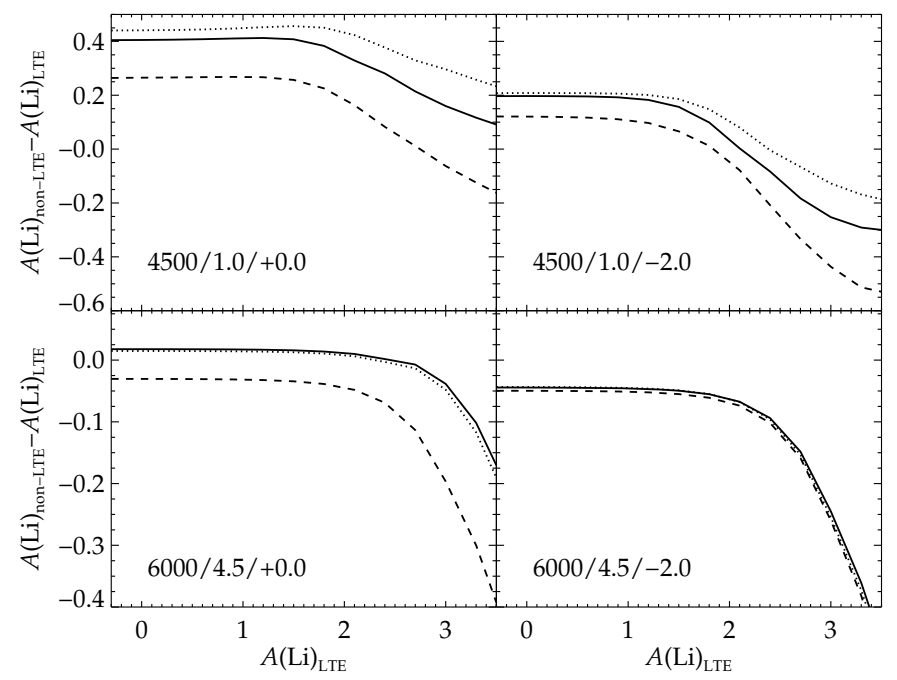

Fig. 2. Panels as in Fig. 1. Solid: same as in Fig. 1. MARCS model atmospheres from Gustafsson et al. (2008) are used, with $\xi_{\mathrm{t}}=2 \mathrm{~km} \mathrm{~s}^{-1}$. Dashed: MARCS model atmospheres from Gustafsson et al. (1975) are used, with $\xi_{\mathrm{t}}=2 \mathrm{~km} \mathrm{~s}^{-1}$. Dotted: MARCS model atmospheres from Gustafsson et al. (2008) are used, with microturbulence parameter set to $\xi_{\mathrm{t}}=5 \mathrm{~km} \mathrm{~s}^{-1}$ in the two upper panels and $\xi_{\mathrm{t}}=1 \mathrm{~km} \mathrm{~s}^{-1}$ in the lower panels.

Non-LTE calculations for lithium in 3D, hydrodynamical model atmospheres have to this date been performed only for a few types of stars (Asplund et al. 2003), and we plan to extend our work to $3 \mathrm{D}$ in the future.

Acknowledgements. We are grateful to Mats Carlsson for his helpful support and comments on this work. K.L. thanks Jon Sundqvist for careful reading of the manuscript. P.S.B. is a Royal Swedish Academy of Sciences Research Fellow supported by a grant from the Knut and Alice Wallenberg Foundation. P.S.B. also acknowledges additional support from the Royal Swedish Academy of Sciences and the Swedish Research Council.

\section{References}

Allen, C. W. 1976, Astrophysical Quantities (London: Athlone), 3rd edition Anstee, S. D., \& O'Mara, B. J. 1995, MNRAS, 276, 859

Asplund, M., Carlsson, M., \& Botnen, A. V. 2003, A\&A, 399, L31

Asplund, M., Lambert, D. L., Nissen, P. E., Primas, F., \& Smith, V. V. 2006, ApJ, 644,229

Barklem, P. S., \& O’Mara, B. J. 1997, MNRAS, 290, 102

Barklem, P. S., Belyaev, A. K., \& Asplund, M. 2003, A\&A, 409, L1

Belyaev, A. K., \& Barklem, P. S. 2003, Phys. Rev. A, 68, 062703

Bonifacio, P., Molaro, P., Sivarani, T., et al. 2007, A\&A, 462, 851

Carlsson, M. 1986, Uppsala Astron. Obs. Rep., 33

Carlsson, M. 1992, in Cool Stars, Stellar Systems, and the Sun, ed. M. S. Giampapa, \& J. A. Bookbinder, ASP Conf. Ser., 26, 499

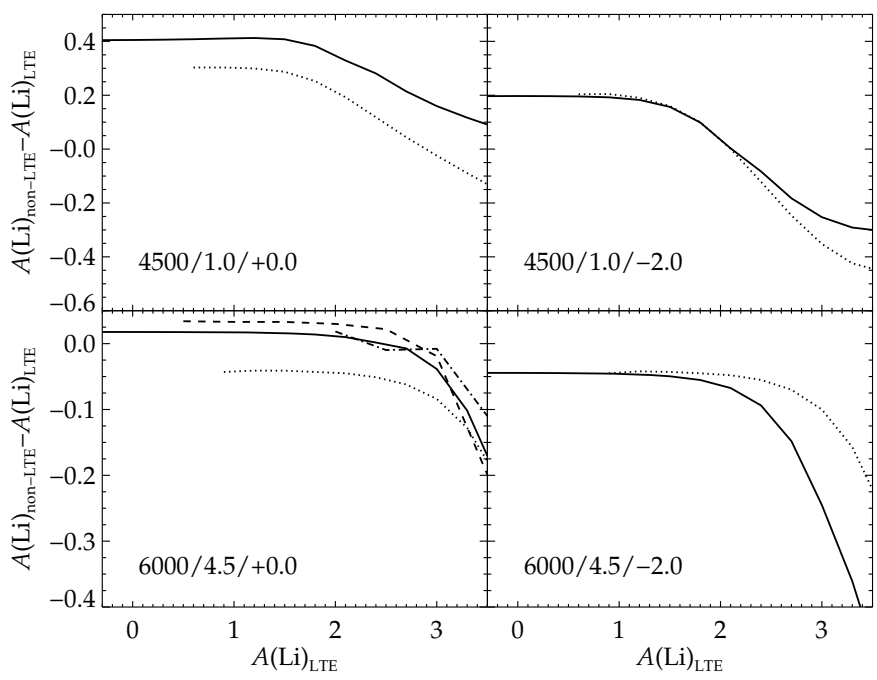

Fig. 3. Panels as in Fig. 1. Solid: same as in Figs. 1 and 2. Dotted: Carlsson et al. (1994) corrections. Dashed: Takeda \& Kawanomoto (2005) corrections. Dash - dotted: Pavlenko \& Magazzu (1996) corrections.

Carlsson, M., Rutten, R. J., Bruls, J. H. M. J., \& Shchukina, N. G. 1994, A\&A, 288,860

Charbonnel, C., \& Primas, F. 2005, A\&A, 442, 961

Croft, H., Dickinson, A. S., \& Gadea, F. X. 1999, MNRAS, 304, 327

Drawin, H.-W. 1968, Z. Phys., 211, 404

Gustafsson, B., Bell, R. A., Eriksson, K., \& Nordlund, A. 1975, A\&A, 42, 407

Gustafsson, B., Edvardsson, B., Eriksson, K., et al. 2008, A\&A, 486, 951

Hosford, A., Ryan, S. G., García Pérez, A. E., Norris, J. E., \& Olive, K. A. 2009, A\&A, 493, 601

Kaulakys, B. 1985, J. Phys. B Atomic Mol. Phys., 18, L167

Korn, A. J., Grundahl, F., Richard, O., et al. 2007, ApJ, 671, 402

Krstić, P. S., \& Schultz, D. R. 2009, J. Phys. B Atomic Mol. Phys., 42, 065207

Kurucz, R. L. 1993, SYNTHE spectrum synthesis programs and line data,

Kurucz CD-ROM, Cambridge, MA: Smithsonian Astrophysical Observatory

Lambert, D. L. 1993, Phys. Scr. T, 47, 186

Lind, K., Primas, F., Charbonnel, C., Grundahl, F., \& Asplund, M. 2009, A\&A, 503,545

Lindgård, A., \& Nielson, S. E. 1977, Atomic Data and Nuclear Data Tables, 19, 533

Park, C. 1971, J. Quant. Spectr. Rad. Trans., 11, 7

Pavlenko, Y. V., \& Magazzu, A. 1996, A\&A, 311, 961

Peach, G., Saraph, H. E., \& Seaton, M. J. 1988, J. Phys. B Atomic Mol. Phys., 21, 3669

Ryan, S. G., Kajino, T., Beers, T. C., et al. 2001, ApJ, 549, 55

Sansonetti, C. J., Richou, B., Engleman, R. J., \& Radziemski, L. J. 1995, Phys. Rev. A, 52, 2682

Seaton, M. J. 1962, Proc. Phys. Soc., 79, 1105

Spite, M., \& Spite, F. 1982, Nature, 297, 483

Steenbock, W., \& Holweger, H. 1984, A\&A, 130, 319

Takeda, Y., \& Kawanomoto, S. 2005, PASJ, 57, 45

Yan, Z.-C., Tambasco, M., \& Drake, G. W. F. 1998, Phys. Rev. A, 57, 1652 